\title{
Study of Bradyarrhythmias in Acute Myocardial Infarction
}

\author{
Samir Rafla $\mathbb{D}^{\circ}$, Sherif Wagdy Ayad ${ }^{\circledR}$, Mohamed Sanhoury $\mathbb{(}$ \\ Department of Cardiology, Faculty of Medicine, Alexandria University, Alexandria, Egypt \\ Email:smrafla@yahoo.com, sherifwagdyayad@yahoo.com,drsanhory@yahoo.com
}

How to cite this paper: Rafla, S., Ayad, S.W. and Sanhoury, M. (2022) Study of Bradyarrhythmias in Acute Myocardial Infarction. World Journal of Cardiovascular Diseases 12, 38-49.

https://doi.org/10.4236/wjcd.2022.121005

Received: December 16, 2021

Accepted: January 23, 2022

Published: January 26, 2022

Copyright $\odot 2022$ by author(s) and Scientific Research Publishing Inc. This work is licensed under the Creative Commons Attribution International License (CC BY 4.0).

http://creativecommons.org/licenses/by/4.0/

\begin{abstract}
Background: Arrhythmias after acute myocardial infarction are common. Bradyarrhythmias need specific insight into when and how to treat them. Objectives: To delineate the incidence, course, and management of different types of bradyarrhythmias after acute myocardial infarction, the study period was five years. Methods: 453 patients with Acute Myocardial Infarction (AMI) were admitted to intensive care in five years. ECGs were analyzed for the presence of bradyarrhythmias and details of management. Results: 65 patients with bradycardia were found. Sinus bradycardia in 40 , sick sinus syndrome in 10 , junctional rhythm in 10, second-degree block in 10, complete heart block in 24 . We divided patients with sinus bradycardia into a stable group and an unstable group. Unstable sinus bradycardia is more prevalent in cases with hypotension or shock, slower heart rates, gross or transmural infarction. Also, predictors of instability were changeable morphology of the "P" wave and inferior rather than anterior infarction. The indications and danger of atropine are defined. Complete heart block was found in 24 patients $(0.053 \%) .13$ were managed by drug therapy (isoprenaline, corticosteroids, and atropine); Eleven patients were paced. 14 out of the 24 patients died (58\%), the total mortality rate among the 453 patients was $22 \%$. The prognostic factors of $\mathrm{CHB}$ were defined. Techniques of introduction of the lead in RV without fluoroscopy are described. Conclusions: Sinus bradycardia in AMI is accompanied by a lower incidence of mortality. Atropine is not a safe drug to be given as routine. Complete heart block predictors of mortality are the association with heart failure, early-onset, and persistence of the block.
\end{abstract}

\section{Keywords}

Acute Myocardial Infarction, Complete Heart Block, Temporary Pacemakers, Bradyarrhythmias, Atropine

\section{Introduction}

Arrhythmias after acute myocardial infarction are common. Bradyarrhythmias 
need specific insight into when and how to treat [1]-[11]. The incidence of each type of bradycardia is variable in different reports as well as the method of management. Thus, we intended to study this important subject and report our findings.

In 1977, the first successful coronary angioplasty was performed by Andreas Gruentzig. Since then, major advances in the management of Myocardial Infarction (MI) were established. Guidelines progressed where primary PCI is now the gold standard therapy. Thrombolytic therapy is also standard if balloon angioplasty is not available in short duration of time. Mortality of MI was reduced by proper intervention and new drugs [12].

\section{Methods}

The study duration was 5 years, 3 years retrospective revision of files of patients who were admitted before (retrospective) and, two years study of patients admitted at the time of the study (prospective). The files of the CCU of the Alexandria Main University Hospital were revised and studied. Of 475 patients admitted, 269 had documented acute myocardial infarction. The remaining patients were either non-coronary cases (admitted for monitoring and intensive observation of their heart condition) or had chest pain that did not prove to be myocardial infarction.

Out of the 269 patients with acute myocardial infarction, 26 had bradyarrhythmias (representing $10 \%$ of cases of AMI).

Patients who were already receiving digoxin or beta-blockers or who revealed bradycardia as an agonal rhythm were not included.

In the next two years; 285 patients were admitted to the CCU; 184 of them had documented AMI. Of these patients, 39 patients had bradyarrhythmias (representing $21 \%$ of Cases of AMI).

The total numbers of admissions to the CCU during 5 years were 775 patients; 453 of them had documented AMI. Of these patients, 65 had different types of bradyarrhythmias listed in Table 1; and the mortality incidence in Table 2.

Some of the patients had more than one type of bradyarrhythmias.

This work has been divided into two parts:

\section{Part I}

263 patients with AMI were admitted, 26 of them had bradyarrhythmias and were included in the study. Of the 26 patients, 18 had sinus bradycardia, 2 junctional rhythms, 2 patients had second degree block and 8 had complete heart block. Some

Table 1. Total patients.

\begin{tabular}{cccc}
\hline & $\begin{array}{c}\text { Total patients } \\
\text { admitted }\end{array}$ & $\begin{array}{c}\text { With } \\
\text { proven MI }\end{array}$ & $\begin{array}{c}\text { With } \\
\text { Bradycardia }\end{array}$ \\
\hline Three years (retrospective study) & 475 & 269 & $26(10 \%)$ \\
Two years (prospective study) & 285 & 184 & $39(21 \%)$ \\
Total & 770 & 453 & $65(14.3 \%)$ \\
\hline
\end{tabular}


Table 2. Incidence of bradyarrhythmias in 453 patients with AMI. The total patients with bradyarrhythmias were 65 .

\begin{tabular}{cccc}
\hline Type & Incidence & Percent (\%) & Mortality \\
\hline Sinus bradycardia & 40 & 9 & $1(2.5 \%)$ \\
Sick sinus syndrome & 10 & 2.2 & - \\
Junctional rhythm & 10 & 2.2 & $1(10 \%)$ \\
Second degree block with bradycardia & 10 & 2.2 & $4(40 \%)$ \\
Complete heart block & 24 & 5.3 & $14(54 \%)$ \\
\hline
\end{tabular}

of the patients showed more than one type of bradycardia.

The data of these patients were analyzed for:

1) Incidence;

2) Time of onset of bradycardia after symptoms;

3) The type of bradycardia;

4) Site of infarction;

5) Associated manifestations;

6) Treatment given;

7) Outcome of each patient.

The files of the patients included (as is the routine of our CCU) a complete history, physical examination, and a 12 lead electrocardiogram recorded on admission and then daily. ECG was monitored continuously using Siemens bedside monitors and central station. Whenever any arrhythmia occurred it was recorded on an ECG strip.

Blood pressure was measured every four hours routinely, but when hypotension, chest pain, or arrhythmias occurred and when drugs were given; the blood pressure was recorded more frequently.

In all patients, the diagnosis of acute myocardial infarction was established by at least two of the following criteria:

1) Typical history of AMI;

2) Sequential (evolutionary) ST-segment and $\mathrm{T}$ wave changes with or without pathological Q;

3) Significant and transient enzyme rises, Bonow et al. [1].

Cases with left bundle branch block, were included only when the symptoms were typical, the enzymes elevated, or when they had an ECG without BBB not more than three weeks before admission.

Ventricular conduction blocks were defined as any of the following: Right Bundle Branch Block (RBBB), Left Bundle Branch Block (LBBB), Left Anterior Hemiblock (LAH), Left Posterior Hemiblock (LPH), or any combination of the above.

In patients who received atropine, its effects on the following items were observed: 1) Heart rate; 2) Blood pressure; 3) Ventricular premature beats; 4) Chest pain; and 5) Electrical conduction. 
The dose and duration of effect were also observed as well as side effects. The master charts present in the files of these patients facilitated revisions of the data needed. It included records of pulse rate, blood pressure, temperature, and drugs given at one time in a chart form. Routine laboratory investigations were done for each patient, blood picture, fasting blood sugar, blood urea, sedimentation rate, enzymes (Troponin, SGOT, and LDH \& SGPT), Blood cholesterol, uric acid, and portable X-ray of the chest.

\section{Part II}

This included all patients with AMI and bradyarrhythmias admitted to the CCU in two years. They were evaluated on the same lines as in part one; in addition, their hospital course was studied.

During this period a total of 285 patients were admitted to the CCU. Out of these patients, 184 had documented AMI, of whom 39 patients revealed bradyarrhythmias. These bradyarrhythmias were as follows: twenty-two had sinus bradycardia. Sixteen had complete heart block, in ten of them temporary pacing was applied (besides one patient in part I), eight had junctional rhythm, and eight revealed second-degree heart block.

In patients with bradycardia, first seen in the reception room outside the CCU, long rhythm ECG strips were obtained to record the number of premature beats before atropine administration, and/or other lines of treatment.

Atropine sulfate was administered in doses of 0.5 to $1 \mathrm{mg}$ intramuscularly or intravenously in most patients with bradycardia including those with atrioventricular block. If the $\mathrm{HR}$ and $\mathrm{BP}$ were not improved the injection was repeated.

In the group with $\mathrm{CHB}$, atropine was used as the initial mode of treatment while arrangements were made for inserting a transvenous temporary pacing catheter.

Isoproterenol (Isuprel) was used only when atropine was ineffective and when no premature contractions were noted. It was stopped before pacemaker insertion.

Dexamethasone (Decadron) was given to patients with $\mathrm{CHB}$ for several days (depending on the duration of the block) in doses of $4 \mathrm{mg}$ I.M. 6 hourly. In patients who were paced antibiotics were given as a prophylactic measure to guard against infection around the pacing catheter.

We also studied a special group of patients who developed a special type of bradycardia that alternates with tachycardia, the so-called "sick sinus syndrome".

The criterion adopted in picking up cases of sick sinus syndrome was persistent sinus bradycardia; especially if associated with the following manifestations:

- Transient and/or negligible effect of atropine on sinus rhythm;

- Atrial fibrillation is transient or permanent especially if associated with a slow ventricular rate;

- Episodes of tachycardia followed by bradycardia or the opposite;

- Cessation of sinus rhythm (sinus arrest);

- Episodes of sinoatrial exit block which are not related to drug therapy, to- 
gether with the presence of atrioventricular conduction disturbances;

- Prolonged Sinus Node Recovery Time (SNRT) whenever feasible in our patients with AMI;

- Follow-up would reveal: a) Very slow sinus bradycardia not responding to atropine or sympathomimetic; b) The occurrence of tachycardia-bradycardia syndrome; or c) Syncopal attacks.

Summary: 453 patients with acute myocardial infarction were admitted to intensive care in five years. ECGs were analyzed for the presence of bradyarrhythmias and analyzed for details of management course during hospitalization and prognosis.

Files of the patients were kept for any future analysis or rechecking of data.

\section{Technique of pacing}

Guiding of the pacing catheter to the right ventricle by the intracardiac electrogram proved to be a very useful method. The procedure was done while the patients were in bed, sparing precious time of transport to the fluoroscopy room. In two obese patients with heart failure and pulmonary congestion, the catheter could not be visualized under the fluoroscopy screen and the intracardiac electrogram was the only method for positioning the catheter. We connect the tip of the pacing catheter to one of the ECG electrodes (of the chest), connected by crocodile clips, thus we can record intracardiac ECG and know when the tip of the pacing catheter enters RV (Figure 1).

The protocol of the study did not include recording echocardiography data or cardiac catheterization results if it was done.

The research protocol was approved by our institutional review board (IRB) and human subject informed consent.

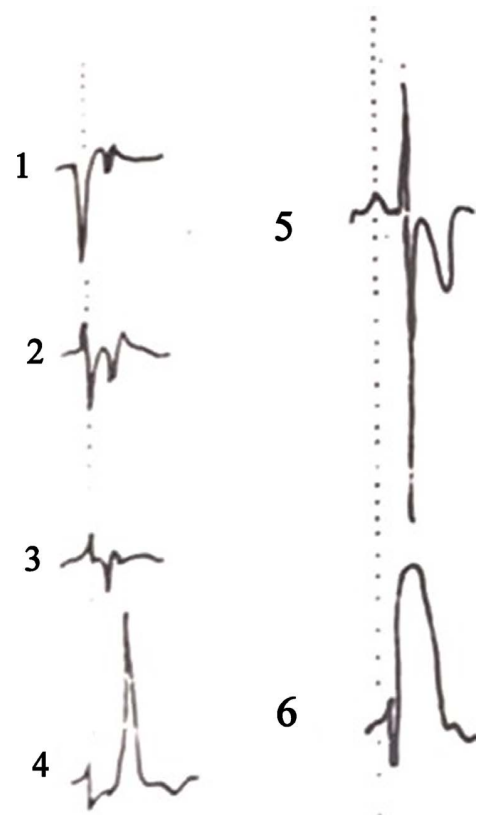

Figure 1. Intracardiac electrocardiograms: In each case, the dotted vertical line indicates the atrial complex (1. High right atrium; 2 . Mid-right atrium; 3. Low right atrium; 4 . Right atrial appendage; 5 . Right ventricle wall; 6 . Left ventricle cavity). 


\section{Statistical analysis of the data}

Data were fed to the computer and analyzed using IBM SPSS software package version 20.0. Qualitative data were described using numbers and percentages. Quantitative data were described using mean and standard deviation. Comparison between different groups regarding categorical variables was tested using the Chisquare test; correlations between two quantitative variables were assessed using the Pearson coefficient. The significance of the obtained results was judged at the 5\% level.

\section{Results}

Out of the 453 patients with acute myocardial infarction, 75 revealed bradyarrhythmias. Ten were excluded because they were receiving digoxin or beta-blockers when first seen. The remaining 65 patients revealed the following types of bradycardia:

40 sinus bradycardia;

10 junctional rhythms;

10 sick sinus syndrome;

10 second degree block;

24 complete heart block.

Some of the patients had had more than one type of bradycardia.

Sinus Bradycardia (SB): The mean age of the 40 patients with suns bradycardia as 59 years, 34 males, 6 females, mortality was in one patient (2.5\%), most patients were discharged after 3 to 9 days. One patient died after 2 hours of hospitalization.

The Site of infarction was inferior in 24 patients, anterior in ten and combined anterior and inferior in three, the size of the infarction was gross in 24 and mild in 16 size of infarction was assessed by the number of leads showing ST elevation.

Hypotension and shock occurred in 10 patients, 7 with inferior infarction, one with anterior and two with combined anterior and inferior infarction.

Heart Failure (HF): None of the patients developed heart failure during the $\mathrm{SB}$, However in 4 patients HF appeared after the extension of the infarction in two and occurrence of new infarction in two patients, SB disappeared ultimately in the 4 patients and manifestations of HF supervened.

Hypotension and shock: Three patients developed hypotension and 7 developed shocks. 6 out of the 7 patients with shock had HR below $50 \mathrm{bpm}$; the mean systolic pressure was raised from $72.5 \mathrm{mmHg}$ to $113 \mathrm{mmHg}$. And the diastolic pressure from $50 \mathrm{mmHg}$ to $74 \mathrm{mmHg}$, this rise was due to the use of atropine with sympathomimetic.

Rhythm disturbances in patients with sinus bradycardia:

VPBs in 9 (26\%), APBs in 6 (15\%), AV blocks in 5 (12,5\%), Junctional rhythm in $8(25 \%)$, ventricular tachycardia in 2, ventricular fibrillation in $3(7.5 \%)$.

No patient developed VT or VF spontaneously during SB. In three patients VF developed after giving atropine with wayamine (levofed, sympathomimetic in two 
patients who were resuscitated successfully and the third after giving $1 \mathrm{mg}$ atropine IV alone, resuscitation failed and the patient died. The dose of atropine first injection was $1 \mathrm{mg}$; as VF developed after it, we suggested in the conclusions that first dose should be less as $0.6 \mathrm{mg}$ (Table 3, Table 4).

Age and sex: There were 18 males with mean age 61.6 years and six females with mean age 53 years.

Mortality in the 24 patients: 14 died (58\%). The 13 patients who were not paced, eight died (61.5\%); of the eleven who were paced six died (54.5\%). In the remaining 5 patients the site of infarction could not be defined because of bundle block.

Site of infarction: Seven patients had inferior infarction with 4 deaths; twelve had anterior infarction with eight deaths (67\%).

Wide QRS: Occurred more in patients with anterior infarction than with inferior infarction (eleven and two patients respectively.

The occurrence of heart failure in $\mathrm{CHB}$ was associated with bad prognosis irrespective of the site of infarction.

Effect of atropine on CHB: Atropine was given to 18 patients with CHB. In 13 no effect of atropine was observed; in 19, ventricular rate increased in 17 of them from a mean of $38 \mathrm{bpm}$ to $52 \mathrm{bpm}$. VPBs occurred after its administration in 3 patients.

Factors that were found to affect the prognosis of these patients: 1) The presence of heart failure; 2) The early onset of block after infarction; 3) The early return of sinus rhythm.

\section{Discussion}

Sinus bradycardia: The incidence of SB in our 453 patients with AMI was 9\%.

Table 3. Comparison between two groups of sinus bradycardia: the "unstable" and the "stable" groups.

\begin{tabular}{lcccc}
\hline & \multicolumn{2}{c}{ Unstable SB } & \multicolumn{2}{c}{ Stable SB } \\
\cline { 2 - 5 } & $(15)$ & $37.5 \%$ & $(25)$ & $62.5 \%$ \\
\cline { 2 - 5 } & No. & $\%$ & No. & $\%$ \\
\hline Hypotension or Shock & 8 & 53.3 & 2 & 8 \\
P. Wave Changeable Morphology & 7 & 47 & 7 & 28 \\
(T or P) Depressed P-R & 1 & & 1 & \\
Size of Infraction: Gross & 12 & 80 & 11 & 44 \\
Size of Infraction: Anterior & - & - & 10 & 40 \\
\multicolumn{1}{c}{$:$ : Inferior } & 12 & 80 & 15 & 60 \\
: Combined & 3 & & - & \\
Heart Rate Below 50/Min: No. & 8 & 53.3 & 9 & 36 \\
Persistent Sinus Bradycardia & 5 & 33.3 & 2 & 8 \\
\hline
\end{tabular}


Table 4. Prognostic factors in 24 patients with acute myocardial infarction and complete heart block.

\begin{tabular}{|c|c|c|c|c|c|}
\hline & No. & $\begin{array}{l}\text { Deaths } \\
(n=14)\end{array}$ & $\begin{array}{c}\text { Alive } \\
(\mathrm{n}=10)\end{array}$ & $x^{2}$ & ${ }^{\mathrm{FE}} \mathbf{p}$ \\
\hline \multicolumn{6}{|l|}{ QRS } \\
\hline$<0.12$ & 7 & $3(42.9 \%)$ & $4(57.1 \%)$ & \multirow{2}{*}{0.974} & \multirow{2}{*}{0.393} \\
\hline$\geq 0.12 \mathrm{Sec}$ & 17 & $11(64.7 \%)$ & $6(35.3 \%)$ & & \\
\hline \multicolumn{6}{|l|}{ Onset of Block } \\
\hline Early & 11 & $10(90.9 \%)$ & $1(9.1 \%)$ & \multirow{2}{*}{$8.866^{*}$} & \multirow{2}{*}{$0.005^{*}$} \\
\hline After 24 Hours & 13 & $4(30.8 \%)$ & $9(69.2 \%)$ & & \\
\hline \multicolumn{6}{|l|}{ Duration of Block } \\
\hline Persistent & 14 & $11(78.6 \%)$ & $3(21.4 \%)$ & \multirow{2}{*}{5.662} & \multirow{2}{*}{$0.035^{\star}$} \\
\hline Sinus Rhythm Returned & 10 & $3(30.0 \%)$ & $7(70.0 \%)$ & & \\
\hline \multicolumn{6}{|l|}{ Pacing } \\
\hline Paced & 11 & $6(54.5 \%)$ & $5(45.5 \%)$ & \multirow{2}{*}{0.120} & \multirow{2}{*}{1.000} \\
\hline Not Paced & 13 & $8(61.5 \%)$ & $5(38.5 \%)$ & & \\
\hline \multicolumn{6}{|l|}{ Heart Failure } \\
\hline With HF & 15 & $13(86.7 \%)$ & $2(13.3 \%)$ & \multirow{2}{*}{$13.211^{\star}$} & \multirow{2}{*}{$<0.001^{\star}$} \\
\hline Without HF & 9 & $1(11.1 \%)$ & $8(88.9 \%)$ & & \\
\hline \multicolumn{6}{|l|}{ Stokes Adams Attacks } \\
\hline Present & 17 & $11(64.7 \%)$ & $6(35.3 \%)$ & \multirow{2}{*}{0.974} & \multirow{2}{*}{0.393} \\
\hline Absent & 7 & $3(42.9 \%)$ & $4(57.1 \%)$ & & \\
\hline
\end{tabular}

$\chi^{2}$ : Chi-square test; FE: fisher exact; $\mathrm{p}: \mathrm{p}$ value for comparing between the studied groups. ${ }^{\star}$ : Statistically significant at $\mathrm{p} \leq 0.05$.

This incidence is lower than that reported by Rotman 1972 [2], the lower incidence in our series may be due to the delay in hospitalization and that we excluded bradycardia in patients taking beta-blockers or digoxin.

The mortality in patients with SB was $2.5 \%$ even though 24 out of the 40 patients had transmural MI. Therefore, SB favors a better outlook but it does not denote a small infarction.

Chadda et al. [4] studied Bradycardia-hypotension syndrome in acute myocardial infarction. They found sixty-eight (17 percent) of 380 patients with acute myocardial infarction had the bradycardia-hypotension syndrome (ventricular rate below 60/min and systolic blood pressure less than $100 \mathrm{~mm} \mathrm{Hg}$ ) during the first 24 hours of admission. They reported that in 61 of the 68 patients, the administration of atropine significantly increased the heart rate (from $46 \pm 14$ to 79 $\pm 12 / \mathrm{min})(\mathrm{p}<0.01)$ and systolic blood pressure (from $70 \pm 15$ to $105 \pm 13 \mathrm{~mm}$ $\mathrm{Hg})(\mathrm{p}<0.001)$. In 26 of the 68 patients, ventricular premature complexes decreased from $9.4 \pm 3 / \mathrm{min}$ to $2.4 \pm 0.7 / \mathrm{min}(\mathrm{p}<0.001)$ after the administration of 
atropine. It is concluded that the bradycardia-hypotension syndrome is not an uncommon complication following acute myocardial infarction and that selected doses of atropine may have a beneficial effect without significant complications.

\section{Electrical instability in SB}

We divided sinus bradycardia into two groups, stable and unstable: Those associated with VPBs, more hypotension or shock, heart failure, are classified as unstable. We found P wave in the ECG bifid or different in morphology denoting atrial infarction or electrical instability in the atrial myocardium, we postulated that these cases are the type that needs intervention with atropine with or without sympathomimetic.

Our results show that VF with sinus bradycardia did not occur but three patients developed VF after correction of SB. The first developed VF after $1 \mathrm{mg}$ atropine IV, pulse rose from 60 to $130 \mathrm{bpm}$ then VF occurred. The other two were given atropine plus wayamine (sympathomimetic to raise blood pressure). VF appeared only after abolishing the vagal tone. So, our results dictate to be very cautious and slow in abolishing SB. Atropine now is advised to start by $0.6 \mathrm{mg}$ instead of $1 \mathrm{mg}$.

The two most frequent bradycardic rhythms in the setting of AMI are sinus bradycardia, seen in $30 \%$ to $40 \%$ of patients [10] [11], and atrioventricular (AV) block, seen in $4 \%$ to $20 \%$ of patients with the following occurrence: $8 \%$ to $15 \%$ first-degree, $5 \%$ to $12 \%$ second-degree, and $6 \%$ to $8 \%$ third-degree AV block [6] [7] [8]. Most of these arrhythmias are associated with inferior AMI [4]-[10]. The incidence of bradyarrhythmias has decreased in the era of thrombolysis and early invasive revascularization [13]-[22].

Junctional bradycardia is an AV nodal area escape rhythm seen in up to $20 \%$ of the patients with AMI when the SA node fails to generate an impulse or there is a complete heart block and has a typical rate of 40 to $60 \mathrm{bpm}$.

Idioventricular rhythm: With the failure of the dominant and subsidiary pacemaker, an escape rhythm at a rate of 30 since we could obtain improvement in the hemodynamics after pacing.

Temporary pacing is indicated prophylactically in patients at risk of developing higher degree AV block, severe sinus node dysfunction, or asystole in acute myocardial infarction [2].

Meine et al. identified inferior MI, older age, worse Killip class at presentation, female sex, current smoking, hypertension, and diabetes as independent predictors of AV block [9].

Of the eleven who were temporarily paced, five had heart failure four had hypotension or shock, seven had Stokes Adams attacks and seven had wide QRS. It is safer to proceed to pace in all patients with $\mathrm{CHB}$ after AMI.

\section{Conclusions}

- Sinus bradycardia in acute myocardial infarction is accompanied by a lower incidence of mortality than the general incidence of mortality in acute myo- 
cardial infarction, during the hospital period.

- The absence of ventricular fibrillation during sinus bradycardia potentiates the assumption of the protective effect afforded by the vagus.

- We designated sinus bradycardia as unstable when is associated with hypotension or shock, slower heart rates, gross or transmural infarction, changeable morphology of the $\mathrm{P}$ wave, associated with other types of arrhythmias, dangerous or recurrent, and inferior rather than anterior infarction.

- Atropine is not a safe drug to be given as a routine or without medical supervision. Patients with sinus bradycardia in whom the heart rate is not very slow and with no hypotension, block nor arrhythmia need not be given atropine.

- Generally speaking, when atropine is given for bradyarrhythmias, it is wise to use the intramuscular route and better to start with $0.6 \mathrm{mg}$ and adjust the dose and its frequency according to the response of the individual patient.

- Sinus bradycardia with shock carries a bad prognosis. Treatment with atropine and sympathomimetics might increase ventricular irritability and such a combination must be evaluated. It is wise then to add an antiarrhythmic drug, e.g., phenytoin.

- Junctional rhythm should not be considered benign arrhythmia because in 50\% of these patients; it was associated with other arrhythmias mainly sick sinus syndrome, second-degree heart block, and complete heart block.

- In the group of patients with "sick sinus syndrome"; the following data were found:

* The immediate prognosis was good;

* Temporary pacing or a stand-by pacemaker is necessary.

- Mobitz type II block in acute myocardial infarction frequently proceeds to complete heart block and has a bad prognosis.

- Complete heart block after acute myocardial infarction carries a high mortality. Some factors influence the prognosis of these patients: a) The presence of heart failure; b) The early onset of block after infarction; c) The return of sinus rhythm improves the prognosis.

- It is safer to proceed for pacing all patients with complete heart block after acute myocardial infarction since pacing improves the hemodynamics and overcomes possible sudden cardiac standstill.

\section{Conflicts of Interest}

The authors declare no conflicts of interest regarding the publication of this paper.

\section{References}

[1] Bonow, R.O., Mann, D.L., Zipes, D.P. and Libby, P. (2011) Braunwald's Heart Disease: A Textbook of Cardiovascular Medicine. 9th Edition, Elsevier Science, Philadelphia.

[2] Rotman, M., Wagner, G.S. and Wallace, A.G. (1972) Bradyarrhythmias in Acute Myocardial Infarction. Circulation, 45, 703-722. https://doi.org/10.1161/01.CIR.45.3.703 
[3] Hans-Joachim, T. (2010) Tachyarrhythmias, Bradyarrhythmias, and Acute Coronary Syndromes. Journal of Emergencies, Trauma, and Shock, 3, 137-142. https://doi.org/10.4103/0974-2700.62112

[4] Chadda, K.D., Lichstein, E., Gupta, P.K. and Choy, R. (1975) Bradycardia-Hypotension Syndrome in Acute Myocardial Infarction. Clinical Study, 59, 158-164. https://doi.org/10.1016/0002-9343(75)90349-6

[5] Davis, W.T., Montrief, T., Koyfman, A. and Long, B. (2019) Dysrhythmias and Heart Failure Complicating Acute Myocardial Infarction: An Emergency Medicine Review. The American Journal of Emergency Medicine, 37, 1554-1561. https://doi.org/10.1016/j.ajem.2019.04.047

[6] Harpaz, D., Behar, S., Gottlieb, S., Boyko, V., Kishon, Y. and Eldar, M. (1999) Complete Atrioventricular Block Complication Acute Myocardial Infarction in the Thrombolytic Era. Journal of the American College of Cardiology, 34, 721-728. https://doi.org/10.1016/S0735-1097(99)00431-3

[7] Harrak, S., Chikhi, F., Fellat, B. and Cherti, M. (2020) Atrioventricular Block in Acute Coronary Syndromes. ARC Journal of Cardiology, 6, 1-6. https://doi.org/10.20431/2455-5991.0602001

[8] Aufderheide, T.P. (1998) Arrhythmias Associated with Acute Myocardial Infarction and Thrombolysis. Emergency Medicine Clinics of North America, 16, 583-600. https://doi.org/10.1016/S0733-8627(05)70019-5

[9] Meine, T.J., Al-Khatib, S.M., Alexander, J.H., et al. (2005) Incidence, Predictors, and Outcomes of High-Degree Atrioventricular Block Complicating Acute Myocardial Infarction Treated with Thrombolytic Therapy. American Heart Journal, 149, 670-674. https://doi.org/10.1016/j.ahj.2004.07.035

[10] Brady, W.J. and Harrigan, R.A. (2001) Diagnosis and Management of Bradycardia and Atrioventricular Block Associated with Acute Coronary Ischemia. Emergency Medicine Clinics of North America, 19, 371-384. https://doi.org/10.1016/S0733-8627(05)70189-9

[11] Musat, D.L., Cotiga, D., Pierce, W. and Arshad, A. (2008) Arrhythmias Complicating Acute Myocardial Infarction-Bradyarrhythmias. In: Hong, M.K. and Herzog, E., Eds., Acute Coronary Syndrome, Springer, London, 163-174. https://doi.org/10.1007/978-1-84628-869-2 16

[12] Lee, Z.V. and Hanif, B. (2018) Historical Perspectives on Management of Acute Myocardial Infarction. In: Watson, T.J., Ed., Primary Angioplasty: A Practical Guide, Springer, Berlin, 1-13. https://doi.org/10.1007/978-981-13-1114-7_1

[13] Brignole, M., Auricchio, A., Baron-Esquivias, G., et al. (2013) 2013 ESC Guidelines on Cardiac Pacing and Cardiac Resynchronization Therapy: The Task Force on Cardiac Pacing and Resynchronization Therapy of the European Society of Cardiology (ESC). Developed in Collaboration with the European Heart Rhythm Association (EHRA). European Heart Journal, 34, 2281.

https://doi.org/10.1093/eurheartj/eht150

[14] Abidov, A., Kaluski, E., Hod, H., et al. (2004) Influence of Conduction Disturbances on Clinical Outcome in Patients with Acute Myocardial Infarction Receiving Thrombolysis (Results from the ARGAMI-2 Study). American Journal of Cardiology, 93, 76-80. https://doi.org/10.1016/j.amjcard.2003.09.016

[15] Swart, G., Brady, W.J., DeBehnke, D.J., Ma, O.J. and Aufderheide, T.P. (1999) Acute Myocardial Infarction Complicated by Hemodynamically Unstable Bradyarrhythmia: Prehospital and ED Treatment with Atropine. The American Journal of Emergency Medicine, 17, 647-652 https://doi.org/10.1016/S0735-6757(99)90151-1

[16] Burri, H. and Nicolas, D. (2018) Acute Management of Bradycardia in the Emer- 
gency Setting. Cardiovascular Medicine, 21, 98-104. https://doi.org/10.4414/cvm.2018.00554

[17] Morrison, L.J., Long, J., Vermeulen, M., Schwartz, B., Sawadsky, B., Frank, J., et al. (2008) A Randomized Controlled Feasibility Trial Comparing Safety and Effectiveness of Prehospital Pacing versus Conventional Treatment: "PrePACE". Resuscitation, 76, 341-349. https://doi.org/10.1016/j.resuscitation.2007.08.008

[18] Brignole, M., Auricchio, A., Baron-Esquivias, G., Bordachar, P., Boriani, G., Breithardt, O.A., et al. (2013) 2013 ESC Guidelines on Cardiac Pacing and Cardiac Resynchronization Therapy: The Task Force on Cardiac Pacing and Resynchronization Therapy of the European Society of Cardiology (ESC). Developed in Collaboration with the European Heart Rhythm Association (EHRA). Europace, 15, 1070-1118. https://doi.org/10.1093/europace/eut206

[19] Kusumoto, R.M., Schoenfeld, M.H., Barrett, R.N., Edgerton, J.R., Kenneth, A., Ellenbogen, K.A., et al. (2019) 2018 ACC/AHA/HRS Guideline on the Evaluation and Management of Patients with Bradycardia and Cardiac Conduction Delay. Circulation, 140, e382-e482. https://doi.org/10.1016/j.hrthm.2018.10.036

[20] Rosa, S.A., Timóteo, A.T., Ferreira, L., Carvalho, R., Oliveira, M., Cunha, P., Viveiros, M.A., Portugal, G., Almeida, M.L., Daniel, P. and Ferreira, R.C. (2018) Complete Atrioventricular Block in Acute Coronary Syndrome: Prevalence, Characterisation and Implication on Outcome. European Heart Journal. Acute Cardiovascular Care, 7, 218-223. https://doi.org/10.1177/2048872617716387

[21] Ibanez, B., James, T., Agewall, S., Bucciarelli-Ducci, A., Bueno, H., et al. (2018) 2017 ESC Guidelines for the Management of Acute Myocardial Infarction in Patients Presenting with ST-Segment Elevation. European Heart Journal, 39, 119-177. https://doi.org/10.1093/eurheartj/ehx393

[22] Gorenek, B., Lundqvist, C.B., Brugada, T.J., Camm, A.J., Hindricks, G. and Huber, K. (2014) Cardiac Arrhythmias in Acute Coronary Syndromes: Position Paper from the Joint EHRA, ACCA, and EAPCI Task Force. Europace, 16, 1655-1673.

https://doi.org/10.1093/europace/euu208

\section{Abbreviations}

$\mathrm{CHB}$ = complete heart block; $\mathrm{MI}=$ myocardial infarction; $\mathrm{SB}=$ sinus bradycardia; SSS $=$ sick sinus syndrome. 